\title{
A importância dos Grupos de Gestantes para a emancipação da parturiente: uma revisão de literatura
}

The importance of Groups of Pregnant Women for the Emancipation of the Parturient: A Literature Review

\section{La importancia de los Grupos de Gestantes para la emancipación de la parturienta: revisión de la literatura}

\section{Jessica Gorito Carvalho ${ }^{1}$, Geísa Sereno Velloso da Silva ${ }^{2}$, Tiago Augusto da Silva ${ }^{3}$, Catia Maria Diogo ${ }^{4}$, José Carlos do Nascimento 5}

Como citar esse artigo. Carvalho, JG; da Silva, GSV; da Silva, TA; Diogo, CM; do Nascimento, JC. A importância dos Grupos de Gestantes para a emancipação da parturiente: uma revisão de literatura. Revista Pró-UniverSUS. 2021 Jul./Dez.; 12 (2)SUPLEMENTO:90 - 93.

\section{Resumo}

No que segue, é feita uma revisão de literatura sobre como o grupo de gestantes podem operar no sentido da otimização do cuidado realizado por parte dos profissionais de saúde. Objetiva-se discutir como grupos de gestantes podem ajudar a melhorar a educação das mesmas. Pata tanto, parte-se de pesquisa qualitativa e descritiva, em cima de diversos artigos de pesquisa empírica e teórica de diversos autores. Constata-se que quando a gestante participa de grupos implementados por profissionais de saúde, todos os objetivos da atenção básica são conseguidos de forma mais rápida e eficaz, já que a comunicação, o diálogo, a educação em saúde, as informações compartilhadas sobre as singularidades individuais e regionais, dentre outros pontos, são melhor trabalhados em grupos.

Palavras-chave: Enfermagem; Gravidez; Mortalidade; Parto; Saúde.

\begin{abstract}
In what follows, a literature review is made on how the group of pregnant women can operate in order to optimize the care provided by health professionals. The objective is to discuss how groups of pregnant women can help improve their education. For both, it is based on qualitative and descriptive research, based on several articles of empirical and theoretical research by different authors. It appears that when the pregnant woman participates in groups implemented by health professionals, all the objectives of primary care are achieved more quickly and effectively, since communication, dialogue, health education, information shared about the singularities individual and regional, among other points, are best worked in groups.

Keywords: Nursing. Pregnancy. Mortality. Childbirth. Health.
\end{abstract}

A continuación se realiza una revisión de la literatura sobre cómo puede operar el grupo de gestantes para optimizar la atención brindada por los profesionales de la salud. El objetivo es discutir cómo los grupos de mujeres embarazadas pueden ayudar a mejorar su educación. Para ambos, se basa en una investigación cualitativa y descriptiva, basada en varios artículos de investigación empírica y teórica de diferentes autores. Parece que cuando la gestante participa en grupos implementados por profesionales de la salud, todos los objetivos de la atención primaria se logran de manera más rápida y efectiva, desde la comunicación, el diálogo, la educación en salud, la información compartida sobre las singularidades individual y regional, entre otros puntos, se trabaja mejor en grupo.

Palabras clave: Enfermería; El embarazo; Mortalidad; Parto; Salud.

\section{Afiliação dos autores:}

${ }^{1}$ Acadêmica em Enfermagem da Universidade de Vassouras, Vassouras, RJ, Brasil. ORCID: https://orcid.org/0000-0001-6364-9067

${ }_{2}^{2}$ Mestre em Enfermagem pela UFRJ, Docente do Curso de Enfermagem da Universidade de Vassouras, Vassouras, RJ, Brasil. ORCID: https://orcid.org/0000-0003-0304-8010.

${ }^{3}$ Doutor em Enfermagem pela Escola de Enfermagem Anna Nery (EEAN) da Universidade Federal do Rio de Janeiro (UFRJ). Mestre em Enfermagem pela UNIRIO. Docente do curso de Enfermagem da Universidade de Vassouras. ORCID: https://orcid.org/0000-0001-6870-5101.

${ }^{4}$ Doutora em Ciências pela UFRJ, Docente do Curso de Enfermagem da Universidade de Vassouras, Vassouras, RJ, Brasil. ORCID: https://orcid.org/0000-0002-7000-5961.

${ }^{5}$ Bacharel em Enfermagem e Ciências Biológicas. Tem Especialização em Planejamento e Gestão Ambiental. Mestrado em Ciências Ambientais. Docente do Curso de Enfermagem da Universidade de Vassouras, Vassouras, RJ, Brasil. ORCID: https://orcid.org/0000-0002-4345-2707. 


\section{Introdução}

A gravidez é uma fase de intenso aprendizado e preparação fisiológica e psicológica para o parto e a maternidade, onde profissionais médicos, enfermeiros e gestantes compartilham conhecimentos ${ }^{1}$.

Pesquisas mostram que compartilhar emoções por meio de grupos ajuda as mulheres grávidas a se sentirem mais relaxadas quando descobrem que outras mulheres compartilham as mesmas preocupações e ansiedades ${ }^{2}$. Isso somado ao fato de que a falta de apoio dada as mulheres que mais necessitam de cuidados parecem estar associada à tendência das gestantes em ter comportamentos de risco para sua saúde e a do feto ${ }^{3}$.

Por meio de conversas e discussões, grupos de gestantes possibilitam a troca de conhecimentos e experiências, ajudando profissionais a compreenderem quais as melhores práticas, dada as singularidades das mulheres e do grupo. Possui propriedades terapêuticas e benéficas para a gestante e seus acompanhantes, sendo assim mais uma possibilidade de enfrentamento às mudanças ocasionadas pela gravidez.

As atividades em grupo são importantes para ajudar as pessoas nos momentos de mudança, adaptandose a novas situações, promovendo o crescimento das pessoas e desenvolvendo seus conhecimentos para administrar sua saúde de acordo com as necessidades que enfrentam. Ajuda a conscientizar sobre a maternidade responsável e compreender que os pais podem e devem participar das reuniões ${ }^{4}$.

Em geral, os grupos de gestantes são desenvolvidos com o objetivo de complementar a assistência prestada na consulta e atender as mulheres em todas as situações psicológicas, físicas e sociais. Melhora a adesão das gestantes aos hábitos mais adequados. Reduz a ansiedade, proporciona uma compreensão mais clara das emoções que ocorrem nesse período e possibilita uma aproximação entre os profissionais e os destinatários do cuidado. Além de apoiar um espaço de troca de conhecimentos e experiências entre os participantes ${ }^{5}$.

O objetivo deste artigo é discutir sobre como grupos de gestantes podem ajudar a melhorar a educação das mesmas, haja vista que o grupo tem um propósito intrinsecamente educativo sob o ponto de vista dos aprendizados trazidos pelos profissionais de saúde e pelo compartilhamento de conhecimentos e experiência entre as gestantes.

\section{Metodologia}

A pesquisa é uma revisão de literatura integrativa. Inicialmente, delimitou-se o problema. Logo após, foram feitos levantamentos de dados. Por fim, os mesmos foram analisados e sintetizados orientando-se para apresentação de resultados. Esse tipo de revisão tem sempre análise de pesquisas muito relevantes para ajudar os profissionais de saúde em suas análises e tomadas de decisão, fornecendo síntese sobre os conhecimentos acerca de um determinado tema. Ademais, serve para mostrar fragilidades nas pesquisas existentes, que deve ser solucionada com mais pesquisas empíricas ou teóricas, a depender do problema.

A busca nos bancos de dados foi feita no BVS (Biblioteca Virtual de Saúde), no LILACS, e no BDENF, onde foram usados, em todos eles, os mesmos padrões de buscas. Como critério de inclusão tem os artigos integrais que falem sobre o tema deste trabalho, ou seja, sobre grupos de gestantes focalizando o processo de educação em saúde, com pesquisas publicadas, em português, que estão disponibilizados na internet de forma gratuita. Não entraram amostras como resumos, assim como os que foram encontrados repetidos, em idioma que não o português, já que este trabalho discute as pesquisas feitas unicamente no Brasil. Foram usados doze artigos.

Como descritores, foram usados os seguintes: "grupos", "gestantes", "educação em saúde" e "enfermagem". Os dados foram operacionalizados no LibreOffice Calc mais atual, para apresentar de forma organizada os dados ao pesquisador e possibilitar uma análise mais fluente e organizada, onde a comparação dos referenciais teóricos usados e análise dos resultados pudesse ser feita com eficiência. A análise focou em ver como cada artigo trata sobre a forma em que grupos de gestantes atuam para tornar o processo de gestação mais humanizado e eficiente em todos os seus objetivos.

\section{Resultados e Discussão}

A pesquisa consistiu em 12 artigos que tratavam de atividades grupais para gestantes. Por meio da análise, constatou-se que 9 artigos foram selecionados na base de dados LILACS e que 3 foram selecionadas na base de dados BDEnf.

No que diz respeito às revistas, foram usadas algumas das revistas mais importantes no país. Importante no sentido de que a grande maioria dos autores costumam consulta-las e possuem influência no cenário acadêmico nacional como um todo.

Os artigos usados objetivaram narrar as formas como os grupos de gestantes experienciam os trabalhos, mais especificamente como operam no processo de educação da gestante em relação ao que está passando e ao que está para enfrentar, possibilitando que o faça da melhor maneira possível. Há ênfase na centralidade de um trabalho interdisciplinar. Em relação às metodologias utilizadas, notamos que 9 tiveram o relato de experiência como abordagem metodológica; 2 publicações utilizaram a pesquisa descritiva como 
método, sendo uma de caráter qualitativo e outra quantitativa; apenas 1 publicação tratou de operar como pesquisa documental.

Podemos perceber que a prática educativa mais usada foi a oficina citada com todas as publicações, mas 1 trouxe dados incríveis, pois relataram visitas no domicílio das gestantes no entorno da conformidade para melhorar as práticas. Todo ser humano está naturalmente inserido na sociedade por conta de suas relações6. Conforme afirma Osório7, a relação entre indivíduos dentro de um grupo orienta-se para centralidade de atividades, já que, por definição, grupo se distingue de meras pessoas juntas: tem objetivo comum. A família é a instituição primordial e mais básica e constitui o primeiro grupo que o indivíduo participa; então começam a formar outros grupos, como escola, trabalho. Portanto, é necessário para a caracterização de um grupo que os membros se unam em torno de um interesse comum; deve haver formas afetivas entre os membros e não puro interesse meramente racional. Isso é importante para unir indivíduos, fazendo-os serem mais do que mesos indivíduos soltos, mas um grupo de fato ${ }^{6}$.

O grupo de gestantes é uma forma de implementar a saúde da gestante por meio de processo educativos que tem efeitos em longo prazo, preparando tanto a gestante quanto o pai do bebê ou companheiro para o que está por vir, que então tem a oportunidade de se conhecer, expressar o que sente e suas dúvidas sobre o momento ${ }^{8}$. As práticas educacionais devem permitir que os indivíduos aprendam habilidades eficazes para fazer escolhas inteligentes sobre sua gestação melhorando a sua vida e do seu bebê. O papel do facilitador é central dentro desse contexto, e é realizado pelos profissionais de saúde, tais como médicos, enfermeiros e agentes comunitários ${ }^{9}$. As estratégias usadas nas abordagens citadas nas publicações assumiram diferentes formas, onde jogos estiveram presentes em todos os artigos, seguindo-se pelos recursos de vídeos educativos e pelas aulas. Esses três métodos tiveram prevalência nos relatos de todos os artigos analisados.

A educação para a promoção da saúde ocorre em nível grupal ou individual. É pela troca de experiência que o grupo de gestantes possibilita o processo educativo mais eficaz, onde cada gestante compartilha e ouve de seu colega o que vivenciam e as ideias que tem sobre esta vivência. Aqui, medos são trabalhados de forma mais eficaz, pois cada gestante percebe que o medo ou dúvida que sentem, não é individual, mas um problema mais abrangente que afeta diversas gestantes e não apenas ela. Ao ouvir o relato de experiências e inquietações, toma conhecimento dos fatos que nelas estão presentes naquele momento e ao mesmo tempo recebe informações científicas sobre o fenômeno da gravidez ${ }^{10-11}$.

Neste grupo, o enfermeiro atua como um facilitador, sendo a tarefa mais importante dar as gestantes um contexto de diálogo profícuo para instigar a formação de conhecimentos da gestante sobre o que está passando e como lidar com o processo de gestação (há uma socialização dos conhecimentos inerente a esses grupos de gestantes) e assim contribuir para a combinação dos fatores que dizem, refletem informações científicas $^{7-12-13-14}$. O facilitador deve se preocupar com a transferência de conhecimento de maneira objetiva e simplificada, onde os termos técnicos são substituídos por conceitos e palavras mais simples e de fácil acesso para as gestantes compreenderem ${ }^{15}$. O facilitador tem enorme responsabilidade, já que atua com pessoas muitas vezes carentes em termos de escolaridade e que tem dificuldades para compreender a linguagem padrão e considerada normal dos profissionais de saúde. O facilitador, além de ser um profissional de saúde, deve operar de forma o mais pedagógica possível. Deve saber que é tão importante ouvir e compreender quanto ensinar ${ }^{16}$.

A equipe multiprofissional é indispensável pois apenas com ela é possível dar as gestantes tudo o que precisam para lidar com seus problemas. Ocorre que as gestantes passam por problemas de ordem física, emocional e social. Compreender e acompanhar esses três processos é fundamental nos grupos de gestantes, pois nestes grupos não se trata apenas da saúde física na gestação, mas da saúde mental e de problemas sociais vivenciados e que afetam a gestação ${ }^{11}$. O profissional que pretende prestar assistência humanizada e preparar o casal deve procurar trabalhar e lidar com o afeto, orientar a gestante a prestar atenção ao seu corpo e ao casal. As contribuições do grupo são expressas quase que unanimemente em diferentes artigos.

No grupo de gestantes é importante ampliar o conhecimento da gestante e do companheiro para que tenham a oportunidade de cuidar do bebê, os diferentes posicionamentos do parto, os locais para realizá-lo, as diferentes linhas de pensamento sobre os tópicos escolhidos para, com os quais escolhas conscientes podem ser feitas; não se deve induzir porque o grupo não quer dar receitas prontas, mas construir estratégias junto a gestantes, levando em conta as individualidades de cada uma. Só pensando em como estão se sentindo com o fato de serem mães, muitas vezes mães adolescentes sem preparação emocional alguma, onde muitas vezes o pai do bebê é ausente, é podem fortalecer a sua confiança, para poderem superar o desafio que é a gestação, desafio que é para todas as mães, mas que é ainda mais forte em alguns contextos sociais marcados pela marginalidade ${ }^{11}$.

\section{Conclusão}

O estudo buscou discutir os grupos de gestantes 
como estratégia educativa no campo da saúde, especificamente no que se refere as possibilidades de melhorias na atenção dada as gestantes pelos profissionais de saúde. O que foi feito através da análise e síntese da literatura sobre o assunto.

Um grupo de gestantes é basicamente um lugar indispensável para a comunidade de mulheres grávidas. Porque no dia do encontro, conhecimento e experiência são trocados entre as mulheres e as equipes interdisciplinares participantes por meio de conversas e discussões. Assim, dúvidas, expectativas e desejos que muitas vezes não são respondidos pelo aconselhamento pré-natal são resolvidos durante o desenvolvimento do grupo por meio dessa troca de informações entre as próprias participantes e a equipe responsável pela organização e orientação das discussões acerca dos temas, muitos destes sugeridos pelas próprias gestantes, fruto de suas dúvidas individuais.

As ações educativas que são realizadas entre os grupos de gestantes são de grande valia para as mães conhecerem os diversos assuntos que importam em sua gestação, em sua saúde física e mental. Para as gestantes, a participação nesses grupos é fundamental para se preparar para essa nova etapa da vida materna, principalmente no que diz respeito ao parto e ao recém-nascido, e suscita mais dúvidas que são também respondidas no grupo. Além disso, o interesse dos pais em se envolver mais no grupo aumentou significativamente, a fim de apoiar melhor as mães durante a gravidez. Dessa forma, enfatiza a importância do apoio da família e, principalmente, a importância do aparecimento do pai neste momento, que muda com a vivência da paternidade desde a gestação.

Isso reflete que as mulheres grávidas precisam ser incentivadas e instigadas para participar desses grupos. Com isso, se pode construir laços afetivos e de confiança com os profissionais e tornar suas atividades educacionais um sucesso. Ressalta-se a importância da instrumentação contínua dos profissionais médicos envolvidos tanto no pré-natal quanto no andamento de grupos de gestantes.

\section{Referências}

1. Sanfelice C, Ressel LB, Stumm KE, Pimenta LF. Crenças e práticas do período gestacional: Uma revisão integrativa. Revista Saúde, 2013. [Acesso em 27 de agosto de 2020] 39(2): 35-48. Disponível em: http://periodicos. ufsm.br/revistasaude/article/view/5524.

2. Herbas DTA. Improvisação: experiência com grupo de gestantes. Revista IGT na Rede. [revista em Internet]2014. [Acesso em 14 de Novembro de 2020] 11( 21): 362- 385. Disponível em: http://www.igt.psc. br/ojs/viewarticle.php?id=496\&layout=html

3. Viellas EF, Gama SGN, Carvalho ML, Pinto LW. Fatores associados à agressão física em gestantes e os desfechos negativos no recém- nascido. J. Pediatr. (Rio J.). 2013 janeiro-fevereiro. [Acesso em 12 de setembro de 2020] 89(1): 83-90. Disponível em: http://www.scielo.br/scielo.php?script=sci arttext\&pid=S002175572013000100 013 .
Departamento de Atenção Básica. Atenção ao pré-natal de baixo risco. 1. ed. rev. Brasília: Ministério da Saúde; 2013.

5. Maron LC. Atividade grupal operativa com gestantes e familiares: um relato de experiência. Contexto \& Saúde. 2011; 10(20): 161-8.

6. Zimerman D. A importância dos grupos na saúde, cultura e diversidade. Vínculo, São Paulo. 2007. [Acesso em 9 de dezembro de 2020) 4(4): 1-16. Disponível em http://pepsic.bvsalud.org/scielo.php?script=sci arttext\&pid=S1806-24902007000100002\&lng=pt\&nrm=iso.

7. Hoga LAK, Reberte LM. A experiência de pais participantes de um grupo de educação para saúde no pré-natal. Cienc. enferm. 2010. [Acesso em 5 de novembro de 2020) 16(1): 105-114. Disponível em: http://www.scielo. $\mathrm{cl} / \mathrm{pdf} / \mathrm{cienf} / \mathrm{v} 16 \mathrm{n} 1 /$ art_12.pdf.

8. Duarte SJH, Borges AP, Arruda GL. Ações de enfermagem na educação em saúde no pré-natal: relato de experiência de um projeto de extensão da universidade federal do mato Grosso. Revista de Enfermagem do Centro Oeste Mineiro. 2011 abril-junho. [Acesso em 1 de dezembro de 2020] 1(2): 277-282 Disponível em: http://www.seer.ufsj.edu.br/index.php/ recom/article/viewArticle/13

9. Hoga LAK, Reberte LM. Técnicas corporais em Grupo de Gestantes: a experiência dos participantes. Rev. bras. enferm. Brasilia. 2006. [Acesso em 10 de dezembro de 2020] 3(1): 308-313. Disponível em: http:/www.scielo. br/scielo.php?script=sci arttext\&pid=S0034-71672006000300011\&lng=en

10. Falcone VM. Atuação multiprofissional e a saúde mental de gestantes. Rev. Saúde Pública. São Paulo. 2005. [Acesso em 24 de outubro de 2020) .39(4): 612-618. Disponível em: http://www.scielo.br/pdf/rsp/v39n4/25534. pdf.

11. Santos MR. Curso de orientação à gestação: repercussões nos pais que vivenciam o primeiro ciclo gravídico. Mundo Saúde. 2008. [Acesso em 10 de setembro de 2020] 32(4): 420-429. Disponível em: http://www. saocamilo-sp.br/pdf/mundo_saude/65/02_Curso_baixa.pdf.

12. Zampieri MFM, Gregório FRP, Custódio ZAO, Regis MI, Brasil C. Processo educativo com gestantes e casais grávidos: possibilidade para transformação e reflexão da realidade. Texto contexto - enferm., Florianópolis. 2010. [Acesso em 9 de dezembro de 2020] 19(4): 719-727, Disponível em: http://www.scielo.br/scielo.php?script=sci_arttext\&pid=S0104 07072010000400015\&lng=en\&nrm=iso.

13. Franco ALS. O grupo Maternar... uma experiência com mulheres gestantes e com estudantes da área de saúde - UFBa. Aletheia. 2012 janeiroabril. [acesso em 15 de setembro de 2020] 37(1): 228-234. Disponível em: http://pepsic.bvsalud.org/pdf/aletheia/n37/n37a16.pdf

14. Delfino MRR. O processo de cuidar participante com um grupo de gestantes: repercussões na saúde integral individualcoletiva. Ciênc. saúde coletiva. Santa Catarina. 2004. [Acesso em 23 de novembro de 2020] 9(4): 1057-1066. Disponível em: http://www.scielo.br/pdf/csc/v9n4/a26v9n4.pdf

15. Rolim MO, Moreira TMM, Viana GRO. Curso para gestantes: ação educativa na perspectiva da co-responsabilide. Online Brazilian Journal of Nursing. [revista na internet]. 2006. [Acesso em 15 de dezembro de 2020] 5(3) . Disponível em: https://pesquisa.bvsalud.org/portal/resource/pt/lil489913

16. Duncan BB, Schmidt MI, Giugliani ERJ, Duncan MS, Giugliani C. Medicina ambulatorial: condutas clínicas em atenção primária. 2. ed. Porto Alegre: Artes Médicas; 2000. 